\title{
Giant Right Ventricular Outflow Tract Myxoma Mimics Pulmonary Embolism:
} A Case Report

\author{
Tomomi Nakajima, MD, ${ }^{1}$ Dung The Bui, $M D,{ }^{2}$ Thien Tam Vu, MD, ${ }^{2}$ Dang Hai Nguyen, ${ }^{3}$ \\ Dinh Hoang Nguyen, $\mathrm{MD}, \mathrm{PhD}^{2}$ \\ ${ }^{1}$ Department of Cardiovascular Surgery, Faculty of Medicine, University of Tsukuba, Tsukuba, Japan; \\ ${ }^{2}$ Department of Cardiovascular Surgery, University Medical Center, University of Medicine and Pharmacy at Ho Chi Minh City, Ho \\ Chi Minh City, Vietnam; ${ }^{3}$ Department of Medical Engineering, University of South Florida, Tampa, FL, USA
}

\section{ABSTRACT}

Right ventricular myxoma is very rare, especially its originating from the right ventricular outflow tract (RVOT) and extending to the main pulmonary artery. Here, we report a case of a giant RVOT myxoma, indistinguishable from pulmonary embolism (PE). Although the myxoma is a candidate for urgent surgery, this case satisfied diagnostic criteria for $\mathrm{PE}$ and had no indication for intervention, according to the guideline. The strategy for this mass can be completely different, depending on the diagnosis. Surgical extraction was selected because of atypical clinical course, findings, and nagging debut for neoplasm. Then it made hemodynamic status stable by releasing RVOT obstruction and allowed to reveal the diagnosis as myxoma histopathologically.

\section{INTRODUCTION}

Myxoma is the most common cardiac tumor, while myxoma originating from right ventricle (RV) is rare, ranging from $2 \%$ to $4 \%$ [McAllister 1979; Blondeau 1990]. Among these, myxoma from the right ventricular outflow tract (RVOT) is relatively rare and reported in approximately 30 cases in the last decade [Lacey 2013] and is difficult to diagnose because clinical symptoms and imaging mimic thrombus. Herein, we report a very rare case of a giant pulmonary subvalvular myxoma case extending into the pulmonary artery.

\section{CASE REPORT}

A 68-year-old man complaining of a one-month history of exertional dyspnea was referred to our department for suspicious acute pulmonary embolism (PE). Computed tomography $(\mathrm{CT})$ revealed a giant mass in the $\mathrm{RV}$ and main pulmonary

Received February 6, 2021; accepted February 6, 2021.

Correspondence: Dinh Hoang Nguyen, MD, PhD, Department of Cardiovascular Surgery, University Medical Center, University of Medicine and Pharmacy at Ho Chi Minh City, Ho Chi Minh City, Vietnam (e-mail: dinh.nb@umc.edu.vn).

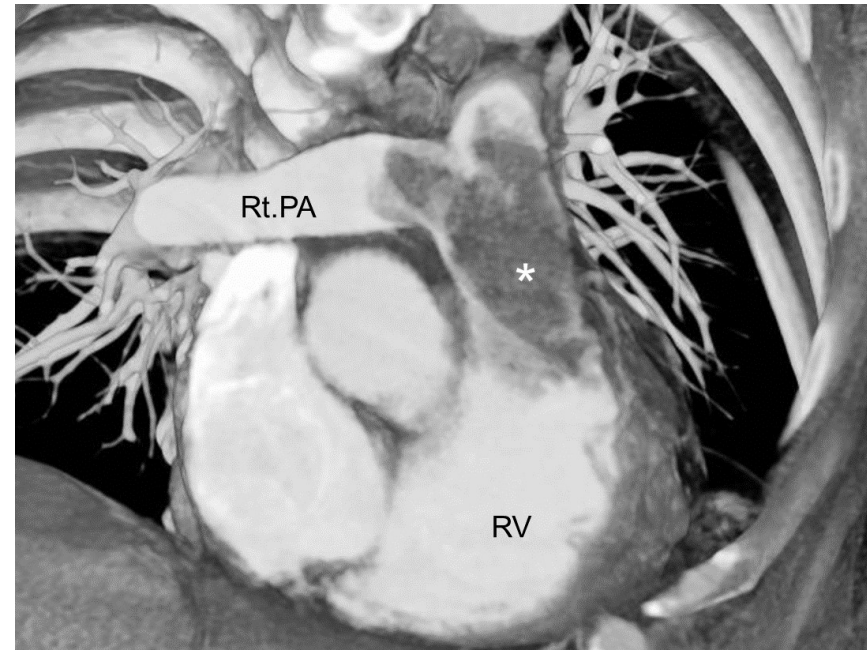

Figure 1. A computed tomographic image showing a giant mass in the main pulmonary artery $\left(^{*}\right)$. Rt. PA, right pulmonary artery; RV, right ventricle

artery. (Figure 1) He had no symptoms without dyspnea, had stable hemodynamic status, but hypoxemia required oxygen inhalation. Echocardiography demonstrated a giant, mobile and hyperechoic mass in the RVOT, suspected RV tumor, and severe tricuspid regurgitation. D-dimer was elevated as 687 $\mathrm{ng} / \mathrm{ml}$ but there was no remarkable change in other indicators. We considered all the circumstances and diagnosed as chronic $\mathrm{PE}$ at moderate risk with differential diagnosis of $\mathrm{RV}$ tumor [Konstantinides 2020]. Even though the guideline of $\mathrm{PE}$ indicated only anticoagulation therapy without any intervention for this patient, the possibility of an $\mathrm{RV}$ tumor could not be denied completely, and we considered to perform surgery not only to confirm its diagnosis or decide long-term therapeutic strategy, but also to stabilize hemodynamic status.

The mass resection was performed urgently under cardiopulmonary bypass and cardiac arrest. Longitudinal pulmonary arteriotomy revealed a dark red, gelatinous tumor occupying the main pulmonary artery, which was continuous from the RV. Right atriotomy was added to ensure that the tumor was not extending into the right atrium and to confirm its localization through the tricuspid valve. The giant tumor originated from the free wall of the RVOT, just below the 


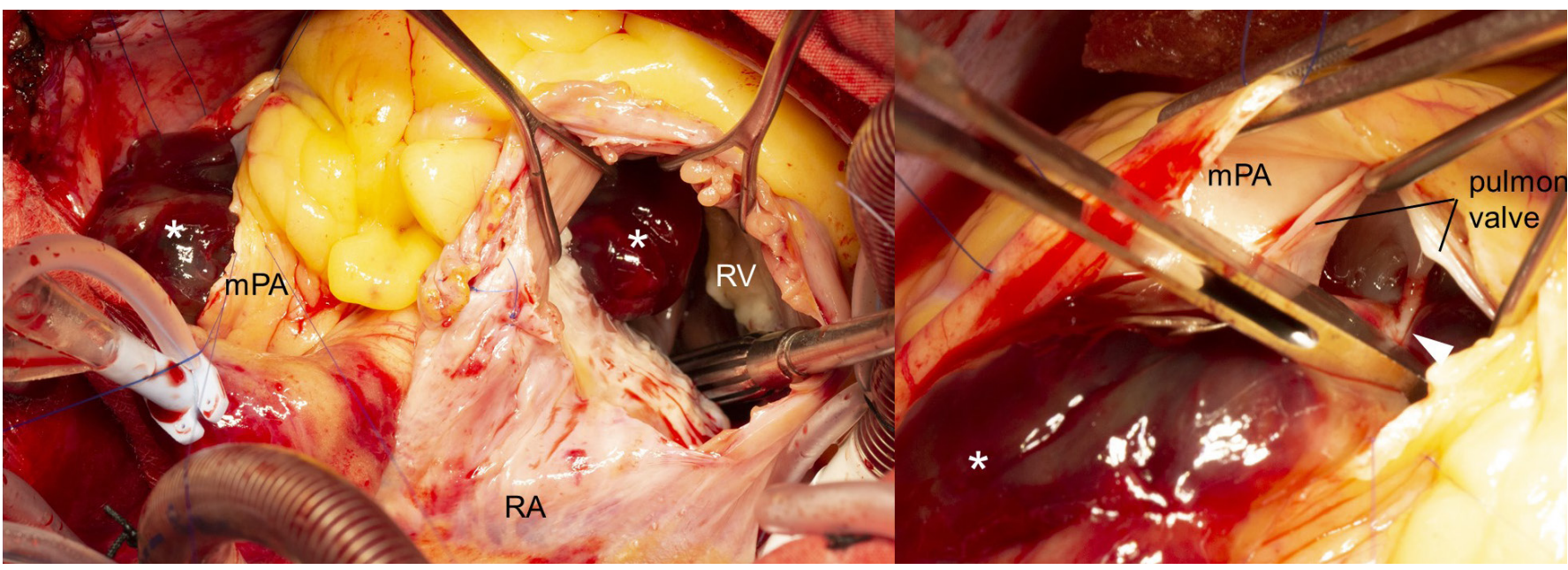

Figure 2. Intraoperative findings. The tumor $(*)$ occupied the right ventricular outflow tract and extended into the main pulmonary artery (A) and attached the free wall of right ventricular outflow tract at just below the pulmonary valve with short stalk (white arrow) (B). mPA, main pulmonary artery; RA, right atrium; RV, right ventricle

pulmonary valve with short stalk, and did not adhere to the pulmonary valve nor the tricuspid valve. (Figure 2) The tumor sized $88 \times 70 \mathrm{~mm}$ was resected en-bloc via the main pulmonary artery. (Figure 3 ) There was no lesion on the trcuspid valve and the anulus was dilated. Intraoperative transesophageal echocardiography showed no residual tumor nor tricuspid regurgitation. Histopathologic findings revealed the diagnosis of myxoma. The postoperative course was uneventful except for secondarily generalized seizures at postoperative day 1 , due to metabolism disorder and discharge occurred on postoperative day 11 .

\section{DISCUSSION}

Although myxoma is the most common benign cardiac tumor usually arising from around the fossa ovalis [Rao 2016], the mass originating from the RVOT and extending across the pulmonary valve into the pulmonary artery is extremely rare with only 6 previously reported myxoma cases to our knowledge [Skillington 1987; Mukadam 1994; Kumagai 2008; Tempe 2010; Ivanovic 2010; Katiyar 2020]. Echocardiography and CT are widely used to diagnose myxoma, however, it is not easy to discriminate myxoma from thrombus or others because the tumor includes a central thrombotic component, avoiding enhancement in CT and letting us recognize as an intracardiac thrombus [Katiyar 2020].

The present case satisfied the diagnostic criteria of PE, according to the guideline, and had no indication for invasive therapy [Konstantinides 2020]. Although the RV tumor was always under consideration, its rarity made the way to true diagnosis more difficult. The mortality of patients awaiting surgery, following definitive diagnosis, has been reported as $8 \%$ in left atrial myxoma [Symbas 1976]. Even though no data has been shown about right-side myxoma, it can cause syncope, dysrhythmia, pulmonary emboli, valvular dysfunction, and sudden death, depending on its position and size [Katiyar 2020; Rao 2016].

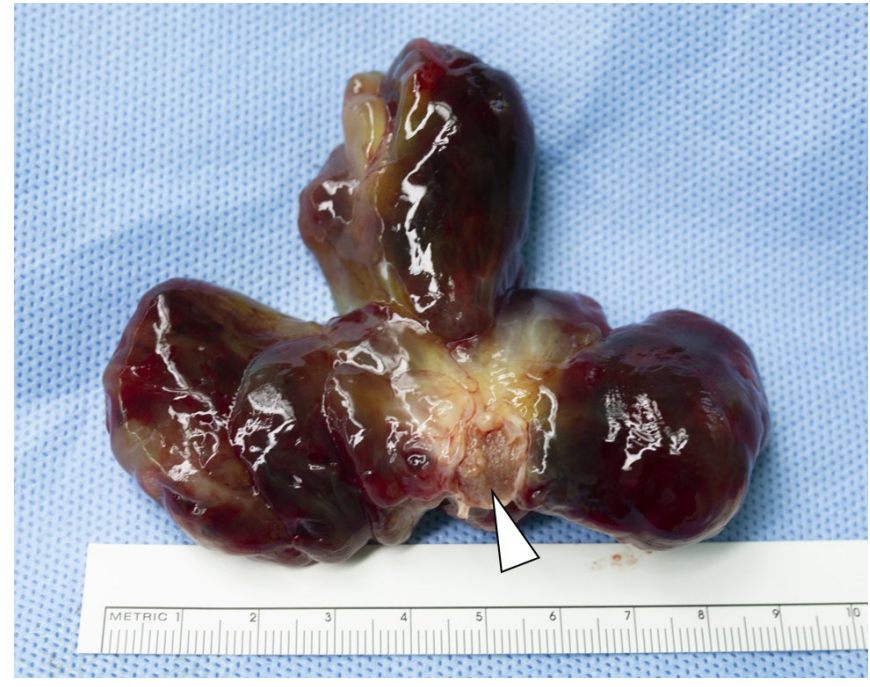

Figure 3. The resected tumor attaching right ventricular outflow tract with short stork (white arrow).

Therefore, surgical excision should urgently be performed. The prompt and accurate diagnosis is important from this aspect, too. Cardiac magnetic resonance imaging is reported to be good at soft tissue contrast resolution and helpful for differential diagnosis [Katiyar 2020], however, it is not always available in any center. It might not be suitable for the urgent diagnosis as considering urgent intervention for acute PE.

In the present case, the clinical condition suggested the chronic state of this mass. The suspicion of a giant thrombus in pulmonary artery without debut history, previous symptoms, nor deep venous thrombosis, pushed the possibility of RV tumor in our mind the whole way. It is important to be aware that some cases diagnosed as chronic or acute PE include neoplastic lesions and to actively suspect other possibilities of diagnosis when the clinical findings are atypical. 


\section{REFERENCES}

Blondeau P. 1990. Primary cardiac tumors-French studies of 533 cases. Thorac Cardiovasc Surg. 38 Sup.2:192-5.

Ivanovic B, Tadic M, Matic D, Simic D. 2010. Right ventricular myxoma obstructing the outflow tract. Am Heart Hosp. J 8:E118-21.

Katiyar G, Vernekar JA, Lawande A, Caculo V. 2020. Cardiac MRI in right ventricular outflow tract myxoma: Case report with review of literature. J Cardiol Cases. 22:128-31.

Konstantinides SV, Meyer G, Becattini C, et al. 2020. 2019 ESC Guidelines for the diagnosis and management of acute pulmonary embolism developed in collaboration with the European Respiratory Society (ERS). Eur Heart J. 21:543-603.

Kumagai K, Sai S, Endo M, Tabayashi K. 2008. Right ventricular myxoma obstructing the pulmonary artery during early childhood. Gen Thorac Cardiovasc Surg. 56:351-3.

Lacey BW, Lin A. 2013. Radiologic evaluation of right ventricular outflow tract myxomas. Tex Heart Inst J. 40:68-70.

McAllister HA Jr. 1979. Primary tumors and cysts of the heart and pericardium. Currm. Probl. Cardiol. 4:1-51.

Mukadam ME, Kulkarni HL, Kumar CJ, Tendolkar AG. 1994. Rightventricular myxoma presenting as right-ventricular outflow-tract obstraction-case report and review of the literature. Thorac Cardiovasc Surg. 42:243-6.

Rao PAS, Prakash SNN, Vasudev S, et al. 2016. A rare case of right ventricular myxoma causing recurrent stroke. Indian Heart J. 68:S97-101.

Skillington PD, Brawn WJ, Edis BD, et al. 1987. Surgical excision of primary cardiac tumors in infancy. Aust N Z J Surg. 57:599-604.

Symbas PN, Hatcher Jr CR, Gravanis MB. 1976. Myxoma of the heart: clinical and experimental observations. Ann Surg. 183:470-5.

Tempe DK, Dutta D, Minhas H, Garg M, Virmani S. 2010. A rare case of myxoma in the right ventricular outflow tract extending to the pulmonary artery. Ann Card Anaesth. 13:167-8. 